\title{
ON THE MEASURABILITY OF VECTOR VALUED FUNCTIONS
}

\author{
KARI ASTALA and KLAUS VALA
}

The English mathematician Percy John Daniell published his now classical paper "A general form of integral" in Texas in 1918. In the same year Carathéorody's work "Vorlesungen über reelle Funktionen" appeared which has perhaps become even more famous. For the theory of measure and integral this epoch signified a definite splitting into two directions: "Le grand écart". Was the integral primarily a functional operation from which the measure of sets had to be derived? Or was it the "geometric" measure of point sets upon which even the theory of integration had to be built, as it was considered by the disciples of Carathéodory?

Nowadays we are quite aware of the fact that this question logically is meaningless: It belongs to the same category of problems as for example the question of deciding by which end to start eating a sausage. Nevertheless, these questions still have their great aesthetic and practical significance.

In the case of vector valued functions the situation is much more complicated than in the scalar case - not merely because of the great variety of different definitions. In this paper we present a Daniell type definition of measurability and show it to be essentially equivalent with the classical definition of Bochner [1] (cf. Corollary 1).

Notations. In the following $X$ is always a set. A subalgebra $\mathscr{H}$ of $\boldsymbol{R}^{X}$ is a clan in $X$ if it is a lattice and has the following property:

$(\sigma)$ There is in $\mathscr{H}$ an increasing sequence $\left(u_{n}\right)$ such that $\lim u_{n}(x)=1$, for all $x \in X$.

A typical representative for a clan would be $\mathscr{K}(X)=\{f \in C(X, \boldsymbol{R}) \mid \operatorname{Supp}(f)$ is compact\}, where $X$ is a locally compact metric space (cf. [4], 12.7.8).

If $\mathscr{H}$ is a clan in $X$ and $\mu$ is a linear form $\mathscr{H} \rightarrow \boldsymbol{R}, \mu$ is said to be a positive measure in $X$ relative to $\mathscr{H}$ if $\mu$ is increasing and satisfies the Daniell condition: $\lim \mu\left(u_{n}\right)=0$ when $\left(u_{n}\right)$ is decreasing and $\lim u_{n}=\overline{0}$ (cf. [2], p. 14).

If $E$ is a vector space, $n \in N, u_{k} \in \mathscr{H}$ and $x_{k} \in E(0 \leqq k \leqq n)$, the condition

$$
t \rightarrow f(t)=\sum_{k=0}^{n} u_{k}(t) x_{k}
$$


defines a mapping $X \rightarrow E$, which we call $\mathscr{H}$-finite. We denote the vectorspace of all these mappings by

$$
\Phi=\Phi(X, \mathscr{H}, E)
$$

Example. Let $C$ be a (Boolean) ring $\subset P(X), \mathscr{H}=\mathscr{H}(C)=\operatorname{sp}\left\{\varphi_{A} \mid A \in C\right\}\left(\varphi_{A}\right.$ is the characteristic function of $A)$. The functions of $\Phi$ are here called $(C, E)$-simple. In this case $f \in \Phi(X, \mathscr{H}, E)$ if and only if $f=\sum_{0}^{n} \varphi_{k} x_{k}$, where $\varphi_{k}=\varphi_{A_{k}}$ and $A_{k} \in C$ $(0 \leqq k \leqq n)$.

Definition. Let $E=(E, \mathscr{T})$ be a topological vector space, $\Phi=\Phi(X, \mathscr{H}, E)$ and $\mu$ a positive measure in $X$ relative to $\mathscr{H}$. We say that a mapping $f: X \rightarrow E$ is $(\mu, \mathscr{H}, \mathscr{T})$-measurable if there exists a sequence $\left(f_{n}\right)$ in $\Phi$ such that $\lim f_{n}=f$ $(\mu$-a.e.) in the sense of the topology $\mathscr{T}$.

If, for example, $E$ is a Banach space, $\mathscr{M}$ is the algebra of $\mu$-measurable sets and $\mathscr{H}=\mathscr{H}(\mathscr{M})$, we get the usual $\mu$-measurability ([3], p. 41).

If $\mathscr{T}$ is weak topology, the corresponding $(\mu, \mathscr{H}, \mathscr{T})$-measurability may be called weak $(\mu, \mathscr{H})$-measurability, and so on.

Lemma. Let $\mathscr{H}$ and $\mathscr{H}^{\prime}$ be clans in $X, \mu: \mathscr{H} \rightarrow \boldsymbol{R}$ and $\mu^{\prime}: \mathscr{H}^{\prime} \rightarrow \boldsymbol{R}$ positive measures in $X$. Suppose each $u \in \mathscr{H}$ is $\mu^{\prime}$-measurable, $\mu^{\prime}(X)<\infty, E$ a vector space, $h \in \Phi(X, \mathscr{H}, E), p$ a seminorm in $E$ and $\varepsilon>0$. Then there exists a $\mu^{\prime}$-measurable $B \subset X$ and a $h^{\prime} \in \Phi\left(X, \mathscr{H}^{\prime}, E\right)$, such that $\mu^{\prime}(B)<\varepsilon$ and $p\left(h^{\prime}(t)-h(t)\right)<\varepsilon$, for $t \in X \backslash B$.

Proof. Let $h(t)=\sum_{k=0}^{n} h_{k}(t) x_{k}$, where $h_{k} \in \mathscr{H}, x_{k} \in E(0 \leqq k \leqq n)$ and $t \in X$. As $h_{k}$ is $\mu^{\prime}$-measurable, there is in $\mathscr{H}^{\prime}$ a sequence $\left(g_{k}^{i}\right)_{i \in N}$, such that $\lim g_{k}^{i}=h_{k}\left(\mu^{\prime}\right.$-a.e. $)$. Because $\mu^{\prime}(X)<\infty$ we can apply Egoroff's theorem ([2], p. 41) and conclude the existence of a $\mu^{\prime}$-measurable $B \subset X$ and $j \in N$, such that $\mu^{\prime}(B)<\varepsilon$ and

for $t \in X \backslash B$. Let

$$
\left|g_{k}^{j}(t)-h_{k}(t)\right|<\frac{1}{(1+n)\left(1+p\left(x_{k}\right)\right)} \varepsilon,
$$

$$
h^{\prime}(t)=\sum_{k=0}^{n} g_{k}^{j}(t) x_{k},
$$

for $t \in X$. Then $h^{\prime} \in \Phi\left(X, \mathscr{H}^{\prime}, E\right)$ and $p\left(h^{\prime}(t)-h(t)\right)<\varepsilon$, for $t \in X \backslash B$.

Theorem. Let $\mu: \mathscr{H} \rightarrow \boldsymbol{R}$ and $\mu^{\prime}: \mathscr{H}^{\prime} \rightarrow \boldsymbol{R}$ be positive measures in $X$. Suppose each $h \in \mathscr{H}$ is $\mu^{\prime}$-measurable and each $\mu$-null set is also $\mu^{\prime}$-null set. Let $(E, \mathscr{T})$ be a locally convex metrizable vector space and $f a(\mu, \mathscr{H}, \mathscr{T})$-measurable function $X \rightarrow E$; then $f$ is $\left(\mu^{\prime}, \mathscr{H}^{\prime}, \mathscr{T}\right)$-measurable.

Proof. The condition $(\sigma)$ implies that $X=\bigcup_{0}^{\infty} A_{n}$, where $A_{n} \subset A_{n+1}$ and $\mu^{\prime}\left(A_{n}\right)<\infty$ for $n \in N$ ([2], p. 40).

Assume $f$ is $(\mu, \mathscr{H}, \mathscr{T})$-measurable. Let $\left(p_{k}\right)_{k \in N}$ be an increasing family of continuous seminorms in $E$ that defines the topology $\mathscr{T}$. There is a sequence $\left(g_{n}\right)_{n \in N}$ 
of functions in $\Phi(X, \mathscr{H}, E)$, such that $g_{n}(t) \rightarrow f(t)$ for $\mu$-almost all $t \in X$. By the above lemma we can find for each $n \in N$ a $\mu^{\prime}$-measurable $B_{n} \subset A_{n}$ and a $g_{n}^{\prime} \in \Phi\left(X, \mathscr{H}^{\prime}, E\right)$, such that $\mu^{\prime}\left(B_{n}\right)<2^{-n}$ and $p_{n}\left(g_{n}(t)-g_{n}^{\prime}(t)\right)<2^{-n}$, for $t \in A_{n} \backslash B_{n}$. Let

$$
\begin{aligned}
& D=\left\{t \in X \mid g_{n}(t)+f(t)\right\}, \\
& B=\varlimsup \lim B_{n}=\bigcap_{n=0}^{\infty} \bigcup_{k=n}^{\infty} B_{k} .
\end{aligned}
$$

Then $\mu(D)=0$, and therefore $\mu^{\prime}(D)=0$. The definition of $B$ implies that $\mu^{\prime}(B)=0$, and so $\mu^{\prime}(D \cup B)=0$. If $t \in X \backslash(D \cup B)$, there is a $n_{0} \in N$, such that $t \notin \bigcup_{k=n_{0}}^{\infty} B_{k}$ and $t \in A_{n}$, for $n>n_{0}$. So $t \in A_{n} \backslash B_{n}$, for $n>n_{0}$, and

$$
\lim _{n \rightarrow \infty} p_{k}\left(g_{n}^{\prime}(t)-f(t)\right) \leqq \lim _{n \rightarrow \infty} p_{k}\left(g_{n}(t)-f(t)\right)+\lim _{n \rightarrow \infty} p_{n}\left(g_{n}^{\prime}(t)-g_{n}(t)\right)=0
$$

for every $k \in N$.

The $\mu$-measurability of a vector valued function is usualy defined by means of the clan formed by simple functions (cf. e.g. [3], p. 41). If the Daniell type approach is employed ([2]), we get immediately from the above theorem the following result (cf. [2], p. 50):

Corollary 1. If $E$ is a normed space, a function $f: X \rightarrow E$ is $\mu$-measurable (in the usual sense) if and only if it is ( $\mu, \mathscr{H},\|\|)$-measurable.

Corollary 2. If $E$ is a normed space, a function $f: X \rightarrow E$ is $(\mu, \mathscr{H},\|\|)-$ measurable if and only if it is weak $(\mu, \mathscr{H})$-measurable.

Proof. Let $f_{n} \in \Phi(X, \mathscr{H}, E)$ and $f_{n}(t) \rightarrow f(t) \mu$-a.e. with respect to the weak topology of $E$. Then $f_{n}(X) \subset E_{n}$, where $E_{n}$ is a finite dimensional subspace of $E$. Let $F$ be the norm closure of the convex hull of $\bigcup_{0}^{\infty} E_{n}$. As $F$ is also weakly closed there is a $\mu$-null set $B \subset X$ such that $f(X \backslash B) \subset F$. Because $E_{n}$ is norm separable for each $n \in N, F$ is norm separable. The numerical function $x^{\prime} \circ f$ is $\mu$-measurable for each $x^{\prime} \in E^{\prime}$. Now the classical Pettis's measurability theorem ([3], p. 42) implies that $f$ is $\mu$-measurable in the usual sense.

Corollary 3. Let $E$ be a normed space and $X, \mu: \mathscr{H} \rightarrow \boldsymbol{R}, \mu^{\prime}: \mathscr{H}^{\prime} \rightarrow \boldsymbol{R}$ as in the above theorem. If then $f: X \rightarrow E$ is weak $(\mu, \mathscr{H})$-measurable it is weak $\left(\mu^{\prime}, \mathscr{H}^{\prime}\right)$ measurable.

The integrability of a $(\mu, \mathscr{H}, T)$-measurable function $f: X \rightarrow E$ is defined exactly in the same manner as the Bochner integrability ([3], p. 44). The simple functions in the latter are now substituted by the functions in $\Phi(X, \mathscr{H}, E)$. The authors intend to return to these questions in some other connexion. 


\section{References}

[1] Bochner, S.: Integration von Funktionen, deren Werte die Elemente eines Vektorraumes sind. Fund. Math. XX, 1933, 262-276.

[2] Descombes, R.: Intégration. - Hermann, Paris, 1972.

[3] Diestel, J., and JR. J. J. Uhl: Vector measures. - Mathematical Surveys, no. 15, American Mathematical Society, Providence, Rhode Island, 1977.

[4] Dieudonné, J.: Éléments d'analyse 2. - Gauthier-Villars, Paris, 1968.

University of Helsinki

Department of Mathematics

SF-00100 Helsinki 10

Finland

Received 22 May 1979 